\title{
ON THE HAUPTVERMUTUNG FOR A CLASS OF OPEN MANIFOLDS $\left({ }^{1}\right)$
}

\author{
BY
}

\author{
A. J. MACHUSKO, JR.
}

1. Introduction. The Hauptvermutung for PL manifolds is the conjecture that homeomorphic PL manifolds are PL homeomorphic. No counterexample to this conjecture is known. It is known to be true for manifolds of dimension three or less ([13], [14]) and for many high dimensional compact manifolds ([20], [21]), but the only high dimensional result for open (i.e. noncompact with empty boundary) manifolds is that it is true in case the manifolds are topologically $E^{n}(n \geqq 5)$ [19].

The main result of this paper is that the conjecture is true whenever the manifolds are topologically $S^{n}$ minus a nonempty tame [8] compact 0 -dimensional subset and $n>5$. The analogous result in the differentiable category holds if $n=6,7$ (Theorem 5.2), but there exist many counterexamples to the complete transfer of the theorem, there is one in dimension 8 . We also obtain a connectivity characterization of $S^{n}$ $(n>5)$ minus a nonempty tame compact 0-dimensional subset (Theorem 4.3).

2. Definitions and basic facts. An end of a manifold $M$ is a function

$$
\varepsilon:\{\text { compact subsets of } M\} \rightarrow\{\text { open subsets of } M\}
$$

such that

(1) $\varepsilon(C)$ is a nonempty component of $M-C$ and

(2) $\varepsilon\left(C_{1}\right) \supset \varepsilon\left(C_{2}\right)$ whenever $C_{1} \subset C_{2}$.

This definition is equivalent to that given by Siebenmann in [16].

Throughout this paper $\mathscr{E} X, \mathscr{C} X, \mathscr{C}_{u}(X-A)$ and $\sigma X$ will denote the set of ends of $X$, the set of components of $X$, the set of unbounded components of $X-A$ (i.e. components with noncompact closure in $X$ ), and the cardinality of $X$ respectively. The following elementary lemma is given without proof.

LemMA 2.1. Let $M^{n}(n \geqq 2)$ be a compact manifold and let $K$ be a 0 -dimensional closed subset of $M$. Then $\sigma \mathscr{E}(M-K)=\sigma K$.

A manifold $M$ is said to be $q$-connected at infinity if and only if given any compact subset $C$ of $M$, there is a compact subset $D$ (depending upon $C$ ) of $M$ such that $C \subset D$ and each component of $M-D$ is $q$-connected. $M$ is $(p, q)$-connected if and only if $M$ is $p$-connected and $q$-connected at infinity.

Received by the editors October 14, 1968.

( $\left.{ }^{1}\right)$ This is a portion of the author's Ph.D. thesis written under the direction of Professor C. H. Edwards, Jr. at the University of Georgia. The work was partially supported by NSF Grant GP-6613. 
Throughout this paper $E^{n}, B^{n}$, and $S^{n}$ will denote the PL manifolds, Euclidean $n$-space, a standard $n$-simplex, and the boundary of $B^{n+1}$ respectively. A cored $n$-ball $C B_{k}^{n}$ of index $k$ ( $k$ a positive integer) is a PL $n$-manifold which is PL homeomorphic to $S^{n}$ minus the interiors of $k$ mutually disjoint PL $n$-balls. $M$ is the monotone union of the submanifolds $M_{1}, M_{2}, \ldots$ if and only if $M=\bigcup M_{i}$ and $M_{i} \subset$ Int $M_{i+1}$ for all $i$.

THEOREM 2.2. If $U$ is an open PL $n$-manifold $(n>5)$ which is the monotone union of cored n-balls, then $U$ is PL homeomorphic to $S^{n}$ minus a nonempty tame compact 0-dimensional subset.

RemarK. We will not give all the details of the proof of Theorem 2.2. The theorem actually can be proven in every dimension except four (in question here because we use the Hauptvermutung for balls). Edwards [6] gives a proof of the 3-dimensional case. One proves Theorem 2.2 in a straightforward manner using the following three lemmas and a theorem of McMillan [8] which tells us that the subset is tame.

LEMMA 2.3. Let $h: M \rightarrow S^{n}$ be a PL embedding where $M$ is a compact connected PL $n$-manifold $(n>5)$ with $\mathrm{Bd} M$ being a disjoint union of $k$ PL $(n-1)$-spheres, $S_{1}, S_{2}, \ldots, S_{k}$. Then $\mathrm{Cl}\left(S^{n}-h(M)\right)$ is the disjoint union of $k$ PL $n$-balls, $B_{1}, \ldots, B_{k}$ with $h\left(S_{i}\right)=\mathrm{Bd} B_{i}$; and hence $M$ is a cored $n$-ball.

Proof. By duality $h\left(S_{i}\right)$ separates $S^{n}$ into two components. One of these, say $Q_{i}$, does not intersect $h(M)$. Since $h\left(S_{i}\right)$ is a PL sphere, $\mathrm{Cl} Q_{i}=B_{i}$ is a topological ball by applying a theorem of Brown [3]. Obviously $B_{i}$ is a component of $\mathrm{Cl}\left(S^{n}-h(M)\right)$ and hence is a PL manifold by [1]. Since for $n>5$ the PL Hauptvermutung for balls is true [18], $B_{i}$ is a PL $n$-ball. The lemma now follows easily.

LEMMA 2.4. Let $C B_{r}^{n} \subset$ Int $C B_{s}^{n}(n>5)$. Let $S_{1}, S_{2}, \ldots, S_{r}$ be the boundary spheres of $C B_{r}^{n}$. Then $\mathrm{Cl}\left(C B_{s}^{n}-C B_{r}^{n}\right)$ has $r$ components, $Q_{1}, \ldots, Q_{r}$ with

$$
Q_{i} \cap C B_{r}^{n}=\operatorname{Bd} Q_{i} \cap \operatorname{Bd} C B_{r}^{n}=S_{i}
$$

and each $Q_{i}$ is a cored $n$-ball.

LEMMA 2.5. Let $M$ be a cored $n$-ball $(n>5)$ with boundary spheres $S_{1}, S_{2}, \ldots, S_{k}$. Let $h: S_{1} \rightarrow S^{n}$ be a $\mathrm{PL}$ embedding such that $\mathrm{Cl} Q$ is a $\mathrm{PL} n$-ball where $Q$ is $a$ component of $S^{n}-h\left(S_{1}\right)$. Let $M^{\prime} \subset \mathrm{Cl} Q$ be a cored $n$-ball with boundary spheres, $S_{1}^{\prime}, S_{2}^{\prime}, \ldots, S_{k}^{\prime}$ and $S_{1}^{\prime}=\mathrm{Bd} \mathrm{Cl} Q$. Then $h$ extends to a PL homeomorphism $h^{\prime}: M \rightarrow M^{\prime}$ such that $h^{\prime}\left(S_{i}\right)=S_{i}^{\prime}$ for $i=1, \ldots, k$.

The PL $n$-manifold $M_{2}$ is said to be obtained from the PL $n$-manifold $M_{1}$ by surgery of index $k(0 \leqq k<n)$ if and only if there are PL embeddings $f_{1}: S^{k} \times B^{n-k} \rightarrow M_{1}$ and $f_{2}: B^{k+1} \times S^{n-k-1} \rightarrow M_{2}$ such that

$$
\begin{aligned}
M_{1} \cap M_{2} & =M_{1}-f_{1}\left(S^{k} \times \operatorname{Int} B^{n-k}\right) \\
& =M_{2}-f_{2}\left(\operatorname{Int} B^{k+1} \times S^{n-k-1}\right),
\end{aligned}
$$


and

$$
f_{1}\left|S^{k} \times S^{n-k-1}=f_{2}\right| S^{k} \times S^{n-k-1} .
$$

Proposition 2.6. Let $f: B^{k+1} \rightarrow M^{n}$ be a proper (i.e. $\left.f^{-1}(\mathrm{Bd} M)=S^{k}\right)$ PL embedding. Then there is a regular neighborhood $N$ of $f\left(B^{k+1}\right)$ in $M$ such that

(1) $M^{\prime}=\mathrm{Cl}(M-N)$ is a PL n-manifold.

(2) If $j<n-k-2$ and $M$ is j-connected, so is $M^{\prime}$.

(3) $N \cap \operatorname{Bd} M \approx S^{k} \times B^{n-k-1}$.

(4) $N \cap \mathrm{Bd} M^{\prime} \approx B^{k+1} \times S^{n-k-2}$.

(5) $\mathrm{Bd} M^{\prime}$ is obtained from $\mathrm{Bd} M$ by surgery of index $k$.

(6) If $2 k+2<n$ and $\lambda$ is the homotopy class of $f \mid S^{k}$ in $\pi_{k}(\mathrm{Bd} M)$, then $\pi_{i}\left(\mathrm{Bd} M^{\prime}\right)$ $\approx \pi_{i}(\mathrm{Bd} M)$ for $i<k$ and $\pi_{k}\left(\mathrm{Bd} M^{\prime}\right) \approx \pi_{k}(\operatorname{Bd} M) /(\lambda)$ where $(\lambda)$ is a subgroup containing $\lambda$.

Proof. Let $N$ be a regular neighborhood of $f\left(B^{k+1}\right)$ in $M$ such that $N \cap \operatorname{Bd} M$ is a regular neighborhood of $f\left(S^{k}\right)$ in $\mathrm{Bd} M$. By [15], there is a block bundle $\xi$ over $f\left(B^{k+1}\right)$ whose total space is $N$ and whose restriction to $f\left(S^{k}\right)$ has total space $N \cap \operatorname{Bd} M$. Also by [15], since $f\left(B^{k+1}\right)$ is collapsible, $\xi$ and $\xi \mid f\left(S^{k}\right)$ are product bundles. Hence, there is a PL homeomorphism $h: B^{k+1} \times B^{n-k-1} \rightarrow N$ such that $h(x, 0)=f(x)$ for all $x \in B^{k+1}$, and $h\left(S^{k} \times B^{n-k-1}\right)=N \cap \mathrm{Bd} M$. Hence (3) is satisfied, and by [1], it follows that (1) is satisfied. Now $M^{\prime}$ and $M-f\left(B^{k+1}\right)$ have the same homotopy type and hence (2) follows easily using standard general position techniques [23]. An elementary point-set argument yields (4), and (5) follows. (6) is an immediate consequence of a theorem of Milnor [10].

The following elementary lemmas are given without proof.

LEMMA 2.7. The complement of a compact subpolyhedron in a connected polyhedron has only finitely many components.

LeMma 2.8. Let $C$ be a compact subset of a connected PL n-manifold $M$. Then $C^{\prime}=C \cup \cup\{Q: Q$ is a bounded component of $M-C$ (i.e. $\mathrm{Cl} Q$ is compact) $\}$ is compact and $M-C^{\prime}$ has only finitely many components.

\section{Approximating open manifolds by compact submanifolds.}

Proposition 3.1. Let $U^{n}(n>5)$ be an open $(0,1)$-connected PL manifold. Let $C$ be a compact subset of $U$ such that each component of $U-C$ is 1-connected and $i: C \subset U$ induces onto homomorphisms $i_{*}: H_{r}(C) \rightarrow H_{r}(U)$ for $r \leqq n-2$.

Then there is a compact connected PL n-submanifold $N$ of $U$ such that

(1) $C \subset$ Int $N$,

(2) $\sigma \mathscr{C} \mathrm{Bd} N=\sigma \mathscr{C}(U-N)=\sigma \mathscr{C}_{u}(U-C)$,

(3) each component of $\mathrm{Bd} N$ and of $U-N$ is 1-connected, and

(4) $i: N \subset U$ induces isomorphisms $i_{*}: H_{r}(N) \rightarrow H_{r}(U)$ for $r \leqq n-3$.

The proof of this proposition parallels closely that of Proposition 4 of [2]. 
For $1 \leqq k \leqq n-3$ let $\mathscr{S}_{k}$ be the statement that there is a compact PL $n$-submanifold $N_{k}$ of $U$ such that (1), (2), and (3) of 3.1 hold if $N_{k}$ replaces $N$, and (4) $i: N_{k} \subset U$ induces isomorphisms $i_{*}: H_{r}\left(N_{k}\right) \rightarrow H_{r}(U)$ for $r \leqq k$ and is onto if $k<r \leqq n-2$.

Let $\mathscr{S}_{k}^{\prime}$ be the statement that if $P$ is a compact subpolyhedron of $U$ with $\operatorname{dim} P$ $\leqq n-2$ and $N_{k}$ is as above then there is a compact connected PL n-submanifold $N_{k}^{\prime}$ of $U$ such that (1), (2), (3) and (4) $)_{k}$ hold if $N_{k}^{\prime}$ replaces $N_{k}, N_{k} \cup P \subset \operatorname{Int} N_{k}^{\prime}$, and $\left(N_{k}^{\prime}-N_{k}\right)$ intersected with any component of $U-N_{k}$ is 1-connected.

The proof will proceed through the following steps.

Step 1. Verify $\mathscr{S}_{1}$.

Step 2. Verify $\mathscr{S}_{2}$.

Step 3. Show that $\mathscr{S}_{k}$ implies $\mathscr{S}_{k}^{\prime}$.

Step 4. Show that $\mathscr{S}_{k}$ implies $\mathscr{S}_{k+1}$ if $2 \leqq k \leqq n-5$.

Step 5. Show that $\mathscr{S}_{n-4}$ implies $\mathscr{S}_{n-3}$.

It is clear that $\mathscr{S}_{n-3}$ implies the proposition.

Step 1. The validity of $\mathscr{S}_{1}$.

Proof. Let $C^{\prime}=C \cup \cup\{Q: Q$ is a bounded component of $U-C\}$. By Lemma $2.8, C^{\prime}$ is compact and $U-C^{\prime}$ is composed of finitely many components, $Q_{1}, \ldots, Q_{r}$, which are precisely the unbounded components of $U-C$. Since $C^{\prime}$ is compact, let $N_{0}^{\prime}$ be a compact PL $n$-submanifold of $U$ such that $C^{\prime} \subset$ Int $N_{0}^{\prime}$. Without loss of generality we assume that $N_{0}^{\prime}$ is connected; for if not, we can join components of $N_{0}^{\prime}$ by arcs, take regular neighborhoods, and let the new $N_{0}^{\prime}$ be the old $N_{0}^{\prime}$ union these neighborhoods.

Now let $a$ and $a^{\prime}$ be the number of components of $\mathrm{Cl}\left(U-N_{0}^{\prime}\right)$ and $U-C^{\prime}$ respectively. By Lemma 2.7, $a$ is finite; and since $C^{\prime} \subset$ Int $N_{0}^{\prime}, a \geqq a^{\prime}$. We must alter $N_{0}^{\prime}$ so that $a=a^{\prime}$. But if $a>a^{\prime}$, then two components $V_{1}$ and $V_{2}$ of $\mathrm{Cl}\left(U-N_{0}^{\prime}\right)$ lie in the same component $Q_{j}$ of $U-C^{\prime}$. Let $\alpha$ be a polygonal arc in $Q_{j}$ such that $\alpha \cap V_{i}=\mathrm{Bd} \alpha \cap \mathrm{Bd} V_{i}=\left\{x_{i}\right\}(i=1,2)$ and Int $\alpha \subset$ Int $N_{0}^{\prime}$. Let $P$ be a regular neighborhood of $\alpha$ in $N_{0}^{\prime}$ such that $P \cap C^{\prime}=\varnothing$. Let $N_{0}^{\prime \prime}=\mathrm{Cl}\left(N_{0}^{\prime}-P\right)$. Since an arc cannot separate $N_{0}^{\prime}$, it now follows that $N_{0}^{\prime \prime}$ is a compact connected PL $n$-submanifold of $U$ such that $C^{\prime} \subset$ Int $N_{0}^{\prime \prime}$ and $\mathrm{Cl}\left(U-N_{0}^{\prime \prime}\right)$ has one less component than $\mathrm{Cl}\left(U-N_{0}^{\prime}\right)$. By finite induction we now assume that $\sigma \mathscr{C} \mathrm{Cl}\left(U-N_{0}^{\prime \prime}\right)=$ $\sigma \mathscr{C}_{u}(U-C)$. But $\sigma \mathscr{C} \mathrm{Cl}\left(U-N_{0}^{\prime \prime}\right)=\sigma \mathscr{C}\left(U-N_{0}^{\prime \prime}\right)$, and hence $N_{0}^{\prime \prime}$ almost satisfies (2). Now let $b$ be the number of components of $\mathrm{Bd} N_{0}^{\prime \prime}$ and $a^{\prime}$ be as above. It follows that $b \geqq a^{\prime}$. If $b>a^{\prime}$, then since $\mathrm{Bd} N_{0}^{\prime \prime}=\mathrm{Bd} \mathrm{Cl}\left(U-N_{0}^{\prime \prime}\right)$, two components $B_{1}$ and $B_{2}$ of $\mathrm{Bd} N_{0}^{\prime \prime}$ are components of $\mathrm{Bd} V$ for some component $V$ of $\mathrm{Cl}\left(U-N_{0}^{\prime \prime}\right)$. Let $\beta$ be a polygonal arc in $V$ such that $\beta \cap \operatorname{Bd} V=\operatorname{Bd} \beta \cap\left(B_{1} \cup B_{2}\right)=\left\{y_{1}, y_{2}\right\}$ with $y_{i} \in B_{i}$. Let $W$ be a regular neighborhood of $\beta$ in $V$. Let $N_{0}=N_{0}^{\prime \prime} \cup P$. Since $\beta$ cannot separate $V$, it is clear, by finite induction, that we may now assume that $N_{0}$ satisfies (1) and (2). Now let $M$ be a component of Bd $N_{0}$. Let $Q$ be the component of $U-C$ which contains $M$. Let $\lambda$ be a generator of $\pi_{1}(M)$. By general position let $f: S^{1} \rightarrow M$ be a PL embedding such that $[f]=\lambda([f]$ is the homotopy class of $f)$. Since $Q$ is 1 -connected extend $f$ to $\bar{f}: B^{2} \rightarrow Q$. By Irwin's embedding theorem we 
assume that $\bar{f}$ is a PL embedding, and putting $\bar{f}$ into general position with respect to $M$ keeping $\bar{f} \mid S^{1}$ fixed, we may assume that $\bar{f}\left(B^{2}\right) \cap M$ is a finite number of PL 1-spheres $S_{1}, S_{2}, \ldots, S_{r}$ with $S_{r}=\bar{f}\left(S^{1}\right)$. Now let $S_{i}$ be an innermost embedded 1-sphere. It follows that $\bar{f}\left(\bar{f}^{-1}\left(S_{i}\right) \cup \operatorname{Int} \bar{f}^{-1}\left(S_{i}\right)\right)$ is a PL properly embedded 2-ball $B_{i}^{2}$ in $N_{0}$ or in a component of $\mathrm{Cl}\left(U-N_{0}\right)$. By taking a regular neighborhood of $B_{i}^{2}$, it follows from Proposition 2.6 that we can replace $N_{0}$ by a compact connected PL $n$-submanifold $N_{1}^{\prime}$ such that the component $M^{\prime}$ of $\mathrm{Bd} N_{1}^{\prime}$ which replaces $M$ by surgery satisfies the condition

$$
\pi_{1}\left(M^{\prime}\right) \approx \pi_{1}(M) /\left(\left[f \mid S_{i}\right]\right)
$$

Continuing in this fashion we may assume that

$$
\pi_{1}\left(M^{\prime}\right) \approx \pi_{1}(M) /\left(\left[f \mid S_{1}\right], \ldots,\left[f \mid S_{r}\right]\right) .
$$

Hence we can kill the generator $\lambda$. Since $\pi_{1}(M)$ is finitely generated, we may assume that $M^{\prime}$ is 1 -connected. By finite induction, we can replace $N_{1}^{\prime}$ by a compact connected PL $n$-submanifold $N_{1}$ such that each component of $\mathrm{Bd} N_{1}$ is 1-connected. Since all alterations occured in $U-C, N_{1}$ satisfies (1). The dimension of the surgery is low so that $N_{1}$ satisfies (2). The fact that $U-N_{1}$ is 1 -connected follows easily by general position or the van Kampen theorem depending upon whether in the inductive stage the new compact submanifold was formed by removing or adding on a regular neighborhood to the old compact submanifold. To verify $(4)_{1}$ consider the exact Mayer-Vietoris sequence for the triad $\left(U ; N_{1}, \mathrm{Cl}\left(U-N_{1}\right)\right)$.

$$
H_{1}\left(\mathrm{Bd} N_{1}\right) \rightarrow H_{1}\left(N_{1}\right) \oplus H_{1}\left(\mathrm{Cl}\left(U-N_{1}\right)\right) \rightarrow H_{1}(U) .
$$

Since each component of $\mathrm{Bd} N_{1}$ is 1-connected, it follows that $i_{*}: H_{1}\left(N_{1}\right) \rightarrow H_{1}(U)$ is an injection. Since $C \subset \operatorname{Int} N_{1}$, using the hypothesis, it follows easily that $i_{*}$ is a surjection.

Step 2. The validity of $\mathscr{S}_{2}$.

Proof. Let $N_{1}$ satisfy $\mathscr{I}_{1}$. We will obtain $N_{2}$ from $N_{1}$. Let $Q_{1}, Q_{2}, \ldots, Q_{s}$ be the components of $\mathrm{Cl}\left(U-N_{1}\right)$. Consider the excision map $\varepsilon:\left(\cup Q_{t}, \cup \operatorname{Bd} Q_{t}\right)$ $\rightarrow\left(U, N_{1}\right)$ and the following commutative diagram with exact rows.

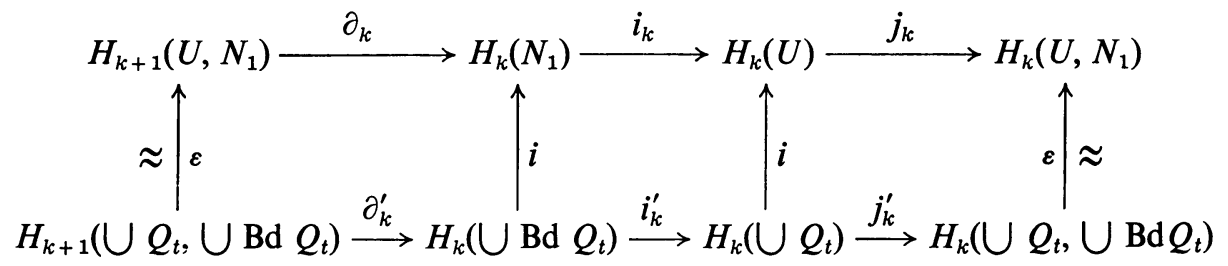

$i_{2}$ is surjective, so we will alter $N_{1}$ to $N_{2}$ so that $N_{2}$ satisfies $\mathscr{S}_{2}$ by killing ker $i_{2}$. Let $0 \neq x \in \operatorname{ker} i_{2}$. Choose $y \in H_{3}\left(U, N_{1}\right)$ such that $\partial_{2}(y)=x$. Hence $z=\partial_{2}^{\prime} \varepsilon^{-1}(y)$ is a nonzero element of $H_{2}\left(\cup \mathrm{Bd} Q_{t}\right)$ such that $i(z)=x$. But $H_{2}\left(\cup \operatorname{Bd} Q_{t}\right) \approx$ 
$\sum H_{2}\left(\mathrm{Bd} Q_{t}\right)$ and hence $z$ can be thought of as $\left(z_{1}, \ldots, z_{s}\right)$ where $z_{t} \in H_{2}\left(\mathrm{Bd} Q_{t}\right)$. Since each Bd $Q_{t}$ is 1 -connected, we represent each $z_{t}$ by a PL embedding $f_{t}: S^{2} \rightarrow \operatorname{Bd} Q_{t}$. But $z \in \operatorname{im} \partial_{2}^{\prime}=\operatorname{ker} i_{2}^{\prime}$ and hence each $\left[f_{t}\right]$ is homologically trivial in $Q_{t}$. Since each $Q_{t}$ is 1-connected, by the Hurewicz theorem and Irwin's embedding theorem, we may extend each $f_{t}$ to a PL embedding $\bar{f}_{t}: B^{3} \rightarrow Q_{t}$. Take regular neighborhoods $P_{t}$ of $\bar{f}_{t}\left(B^{3}\right)$ in $Q_{t}$. By the proof of Proposition 2.6, these are PL balls and $P_{t} \cap \mathrm{Bd} Q_{t} \approx \bar{f}_{t}\left(S^{2}\right) \times B^{n-3}$. Let $N_{2}^{\prime}=N_{1} \cup\left(\cup_{t=1}^{s} P_{t}\right)$. Consider the proper triad $\left(N_{1} \cup P_{1} ; N_{1}, P_{1}\right)$ and the resulting exact Mayer-Vietoris sequence

$$
H_{2}\left(\bar{f}_{1}\left(S^{2}\right) \times B^{n-3}\right) \stackrel{\alpha}{\longrightarrow} H_{2}\left(N_{1}\right) \oplus H_{2}\left(P_{1}\right) \longrightarrow H_{2}\left(N_{1} \cup P_{1}\right) \longrightarrow 0 .
$$

It follows that $H_{2}\left(N_{1}\right) /(\mathrm{im} \alpha) \approx H_{2}\left(N_{1} \cup P_{1}\right)$, i.e.

$$
H_{2}\left(N_{1}\right) /\left(\left[f_{1}\right]\right) \approx H_{2}\left(N_{1} \cup P_{1}\right) \text {. }
$$

Hence $H_{2}\left(N_{2}^{\prime}\right) \approx H_{2}\left(N_{1}\right) /\left(\left[f_{1}\right], \ldots,\left[f_{s}\right]\right) \approx H_{2}\left(N_{1}\right) /(x)$. Since ker $i_{2}$ is finitely generated, by finite induction, there is a compact PL $n$-submanifold $N_{2}$ of $U$ such that $i: N_{2} \subset U$ induces an isomorphism $i_{*}: H_{2}\left(N_{2}\right) \rightarrow H_{2}(U)$. It can easily be checked that $N_{2}$ satisfies (1), (2), (3), and (4)

Step 3. $\mathscr{S}_{k}$ implies $\mathscr{S}_{k}^{\prime}$.

Proof. Let $N_{k}$ satisfy $\mathscr{S}_{k}$. Since $U$ is 1-connected at infinity, let $C^{\prime}$ be a compact subset of $U$ such that $C^{\prime} \supset N_{k} \cup P$ and each component of $U-C^{\prime}$ is 1-connected. Let $N^{\prime}$ be a compact connected PL $n$-submanifold of $U$ which satisfies $\mathscr{S}_{1}$ for the compact subset $C^{\prime}$. Every component of $\mathrm{Cl}\left(U-N^{\prime}\right)$ is a subset of a component of $U-N_{k}$ and hence by an argument like that given in Step 1, we may assume that, in addition, $\sigma \mathscr{C} \mathrm{Cl}\left(U-N^{\prime}\right)=\sigma \mathscr{C}\left(U-N_{k}\right)$. (Note: It is here that we use that fact that $\operatorname{dim} P \leqq n-2$, for recall in Step 1 we join components by arcs; and here we must be sure that the arcs do not intersect $P$, for otherwise it may be that $P \nsubseteq N^{\prime}$.) Now let $N_{k}^{\prime}$ be a PL $n$-submanifold of $U$ which satisfies $\mathscr{S}_{k}$ for the compact set $N^{\prime}$. It is easy to check that $N_{k}^{\prime}$ satisfies $\mathscr{S}_{k}^{\prime}$.

Step 4. $\mathscr{S}_{k}$ implies $\mathscr{S}_{k+1}, 2 \leqq k \leqq n-5$.

Proof. Let $N_{k}$ satisfy $\mathscr{S}_{k}$. Roughly, we wish to alter $N_{k}$ so as to kill $i_{k+1 *}$ where $i: N_{k} \subset U$ and $i_{k+1 *}: H_{k+1}\left(N_{k}\right) \rightarrow H_{k+1}(U)$ without disturbing the other nice properties of $N_{k}$. Let $0 \neq x \in \operatorname{ker} i_{k+1 *}$. Choose $y \in H_{k+2}\left(U, N_{k}\right)$ such that $\partial_{k+2}(y)$ $=x$. Since $\operatorname{dim}($ carrier $y)<n-2$, by Step 3 , choose $N_{k}^{\prime}$ which satisfies $\mathscr{S}_{k}^{\prime}$ for $N_{k} \cup$ carrier $y$.

Let $Q_{1}, Q_{2}, \ldots, Q_{s}$ and $Q_{1}^{\prime}, Q_{2}^{\prime}, \ldots, Q_{s}^{\prime}$ be the components of $\mathrm{Cl}\left(U-N_{k}\right)$ and $\mathrm{Cl}\left(U-N_{k}^{\prime}\right)$ respectively. Let $X_{i}=Q_{i} \cap \mathrm{Cl}\left(N_{k}^{\prime}-N_{k}\right)$. Hence, each $X_{i}$ is 1-connected, $\operatorname{Bd} X_{i}=\operatorname{Bd} Q_{i} \cup \operatorname{Bd} Q_{i}^{\prime}$, and $\mathrm{Bd} Q_{i}, \operatorname{Bd} Q_{i}^{\prime}$ are each 1-connected. Consider the excisions

$$
\varepsilon:\left(\bigcup Q_{i}, \bigcup \operatorname{Bd} Q_{i}\right) \subset\left(U, N_{k}\right), \quad \varepsilon^{\prime}:\left(\bigcup Q_{i}, \bigcup X_{i}\right) \subset\left(U, N_{k}^{\prime}\right)
$$


and the following commutative diagram

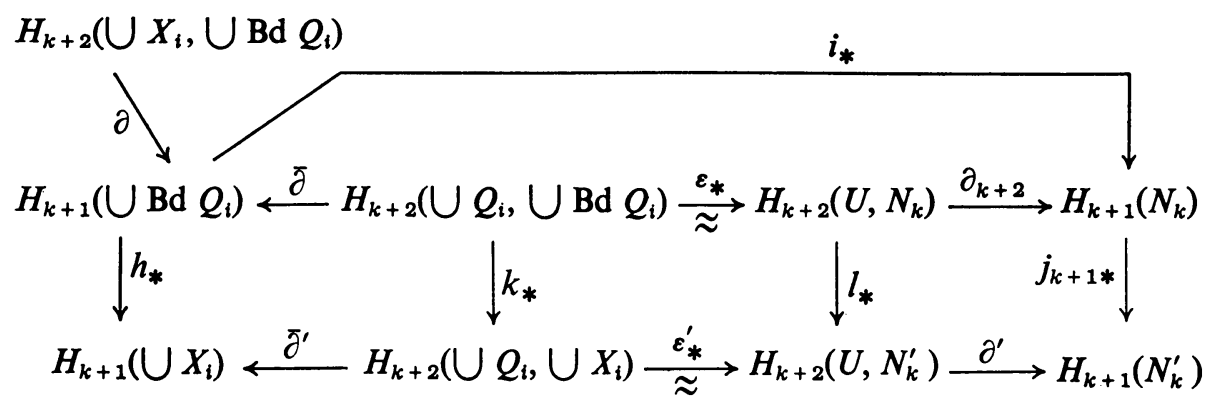

where $i_{*}, h_{*}, k_{*}, l_{*}$, and $j_{k+1 *}$ are all induced by inclusions. Since $N_{k}^{\prime} \supset$ carrier $y$, $l_{*}(y)=0$. Let $z=\bar{\partial}_{\varepsilon_{*}^{-1}}(y)$. Hence $i_{*}(z)=x$ and $h_{*}(z)=0$. Now choose $w \in$ $H_{k+2}\left(\bigcup X_{i}, \bigcup \operatorname{Bd} Q_{i}\right)$ such that $\partial(w)=z$. But $H_{k+1}\left(\bigcup \operatorname{Bd} Q_{i}\right) \approx \sum H_{k+1}\left(\operatorname{Bd} Q_{i}\right)$ and $H_{k+2}\left(\cup X_{i}, \cup \mathrm{Bd} Q_{i}\right) \approx \sum H_{k+2}\left(X_{i}, \mathrm{Bd} Q_{i}\right)$. Hence we may think of $z$ and $w$ as $z=\left(z_{1}, z_{2}, \ldots, z_{s}\right)$ where $z_{i} \in H_{k+1}\left(\mathrm{Bd} Q_{i}\right), w=\left(w_{1}, w_{2}, \ldots, w_{s}\right)$ where

$$
w_{i} \in H_{k+2}\left(X_{i}, \operatorname{Bd} Q_{i}\right) \text {. }
$$

Since each $X_{i}$ and Bd $Q_{i}$ is 1-connected, $\pi_{1}\left(X_{i}, \mathrm{Bd} Q_{i}\right)=0$. Since $i_{*}: H_{r}\left(N_{k}\right) \rightarrow H_{r}(U)$ and $i_{*}: H_{r}\left(N_{k}^{\prime}\right) \rightarrow H_{r}(U)$ are isomorphisms for $r \leqq k$, so is $j_{*}: H_{r}\left(N_{k}\right) \rightarrow H_{r}\left(N_{k}^{\prime}\right)$. Hence $H_{r}\left(N_{k}^{\prime}, N_{k}\right)=0$ for $r \leqq k$. Using excision it follows that $H_{r}\left(X_{i}, \operatorname{Bd} Q_{\imath}\right)=0$ for $1 \leqq i \leqq s$ and $r \leqq k$. By the relative Hurewicz theorem, $\pi_{r}\left(X_{i}, \operatorname{Bd} Q_{i}\right)=0$ for $r \leqq k$; and hence by Lemma 8 of [2], each $w_{\imath}$ can be represented by an embedded handle $B_{i}^{k+2} \times B_{i}^{n-k-2}$ meeting Bd $X_{i}$ in $S_{i}^{k+1} \times B_{i}^{n-k-2} \subset \mathrm{Bd} Q_{i}$.

Let $N_{k+1}=N_{k} \cup \bigcup_{i=1}^{s}\left(B_{i}^{k+2} \times B_{i}^{n-k-2}\right)$. It follows that $x=i_{*}(z)=i_{*}(\partial w)$ includes trivially in $H_{k+1}\left(N_{k+1}\right)$, where we recall that $x$ is an arbitrary generator of ker $i_{k+1 *}$. The desired result will follow by finite induction if we do not change any of the nice properties of $N_{k}$. By finite induction, we may assume that $N_{k+1}=N_{k} \cup B^{n}$ with $B^{n} \cap N_{k}=S^{k+1} \times B^{n-k-2}$. Considering the Mayer-Vietoris sequence for the triad $\left(N_{k+1}, N_{k}, B^{n}\right)$, one concludes that $N_{k} \subset N_{k+1}$ induces homology isomorphisms through dimension $k$, and since $N_{k} \subset U$ induces homology isomorphisms through dimension $k$; it follows that $N_{k+1} \subset U$ induces homology isomorphisms through dimension $k$. Furthermore, it is easy to see that $H_{k+1}\left(N_{k+1}\right)$ is being reduced so that eventually we will kill ker $i_{k+1 *}$. It now follows easily that $N_{k+1}$ satisfies (1), (2) and (4) $)_{k+1}$ of $\mathscr{S}_{k+1}$. We will check (3). Let $\dot{Q}^{\prime}$ be a component of Bd $N_{k+1}$. It follows that $\dot{Q}^{\prime}$ is obtained from a component $\dot{Q}$ of $\mathrm{Bd} N_{k}$ by $(k+1)$-dimensional surgery. Hence

$$
\dot{Q}^{\prime}=\left(\dot{Q}-\left(S^{k+1} \times \operatorname{Int} B^{n-k-2}\right)\right) \cup\left(B^{k+2} \times S^{n-k-3}\right)=C \cup D
$$

where $C \cap D=S^{k+1} \times S^{n-k-3}$. But $C$ has the homotopy type of $\dot{Q}-S^{k+1}$ and $k+1 \leqq n-4$. Hence, by general position, since $Q$ is 1 -connected, so is $C$. Since $\min \{k+1, n-k-3\} \geqq 2$, by the van Kampen theorem it now follows that $\dot{Q}^{\prime}$ is 
1-connected. Let $Q^{\prime}$ be a component of $\mathrm{Cl}\left(U-N_{k+1}\right)$. Let $Q$ be the component of $\mathrm{Cl}\left(U-N_{k}\right)$ such that $Q^{\prime}=\mathrm{Cl}\left(Q-\left(B^{k+2} \times B^{n-k-2}\right)\right)$. Since $Q$ is 1 -connected and $Q^{\prime}$ has the homotopy type of $Q-B^{k+2}$, by general position $Q^{\prime}$ is 1 -connected.

Step 5. $\mathscr{S}_{n-4}$ implies $\mathscr{S}_{n-3}$.

Proof. Let $N$ satisfy $\mathscr{S}_{n-4}$ and let $V=\mathrm{Cl}(U-N)$. Consider the excision map $\varepsilon:(V, \mathrm{Bd} N) \subset(U, N)$ and the following commutative diagram

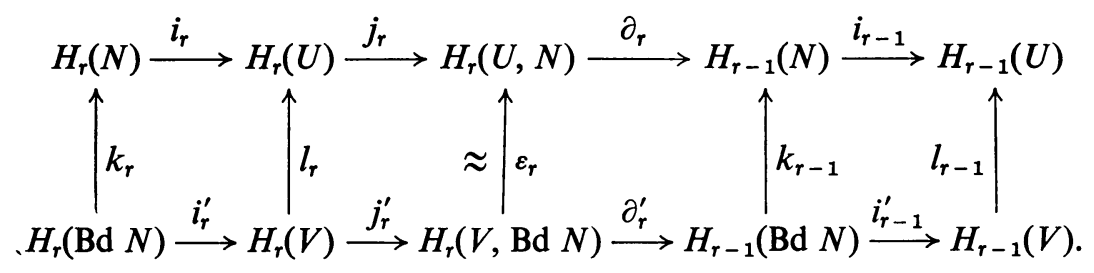

For $r \leqq n-2, i_{r}$ is onto, and hence $i_{r}=0$ and $\partial_{r}$ is injective. For $r-1 \leqq n-4, i_{r-1}$ is an isomorphism and hence $\partial_{r}=0$ for $r \leqq n-3$. Hence $H_{r}(U, N)=0=H_{r}(V, \mathrm{Bd} N)$ for $r \leqq n-3$. Since each component of $\mathrm{Bd} N$ is 1-connected, by duality and the Universal Coefficient theorem, it follows that $H_{n-3}(\mathrm{Bd} N)$ is free. But $j_{n-2}=0$ implies that $j_{n-2}^{\prime}=0$, and hence $\partial_{n-2}^{\prime}$ is injective. So $H_{n-2}(V, \mathrm{Bd} N)$ and $H_{n-2}(U, N)$ are both free. Now consider the following portion of the above diagram

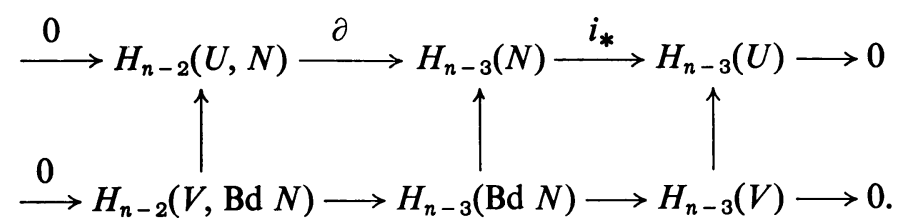

We wish to kill ker $i_{*}$. Since $N$ is compact, let $\lambda_{1}, \lambda_{2}, \ldots, \lambda_{s}$ be a set of generators of ker $i_{*}$. Choose $(n-2)$-chains $c_{1}, \ldots, c_{s}$ in $U$ such that $\partial\left(c_{i}\right)=\lambda_{i}$. Let $P=\bigcup$ carrier $c_{i}$. By Step 3, let $N^{\prime}$ satisfy the conclusion of $\mathscr{S}_{n-4}^{\prime}$ relative to $N$ and $P$. Since $N^{\prime}$ satisfies the same homology conditions as that of $N$, the conclusions we made above for $N$ and $V$ are valid for $N^{\prime}$ and $V^{\prime}=\mathrm{Cl}\left(U-N^{\prime}\right)$. Now consider the commutative diagram with exact rows

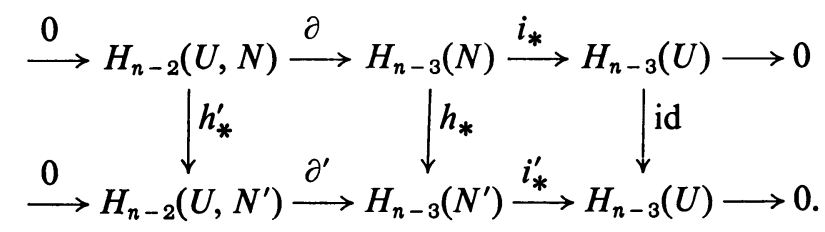

Since Int $N^{\prime} \supset \bigcup$ carrier $\lambda_{i}, h_{*}\left(\operatorname{ker} i_{*}\right)=0$. Hence $h_{*}^{\prime}=0$. Let $X=\mathrm{Cl}\left(N^{\prime}-N\right)$. Since $(V, \operatorname{Bd} N) \subset(U, N)$ and $(V, X) \subset\left(U, N^{\prime}\right)$ are excisions; it follows that $\bar{h}:(V, \operatorname{Bd} N) \subset(V, X)$ induces the 0 map $\bar{h}_{*}: H_{n-2}(V, \operatorname{Bd} N) \rightarrow H_{n-2}(V, X)$. Also $H_{r}(V, X)=0$ for $r \leqq n-3$, and $H_{n-2}(V, X)$ is free. Now since $H_{n-2}(V, X)$ and $H_{n-2}(V, \operatorname{Bd} N)$ are both free, using the Universal Coefficient theorem, it follows 
that $\bar{h}^{*}=0$. Consider the following commutative diagram with exact rows where all vertical homomorphisms are induced by inclusions

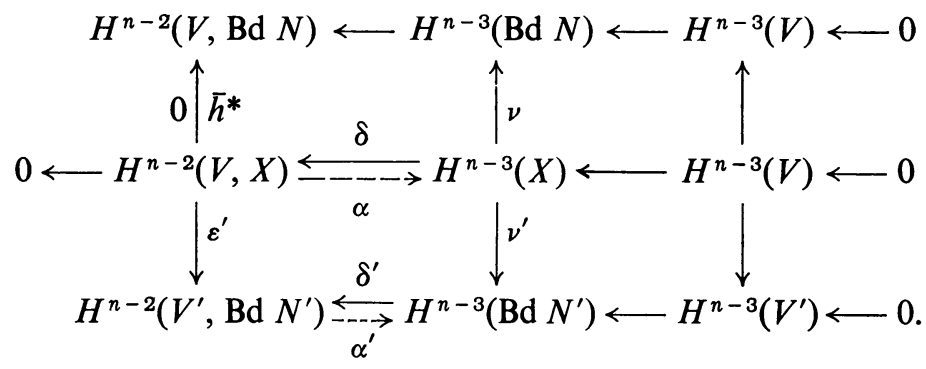

The zeros on the right are valid by the Universal Coefficient theorem since $H_{r}(V, \mathrm{Bd} N)=H_{r}(V, X)=H_{r}\left(V^{\prime}, \mathrm{Bd} N^{\prime}\right)=0$ for $r \leqq n-3$. To show that $\delta$ is onto, consider the commutative diagram

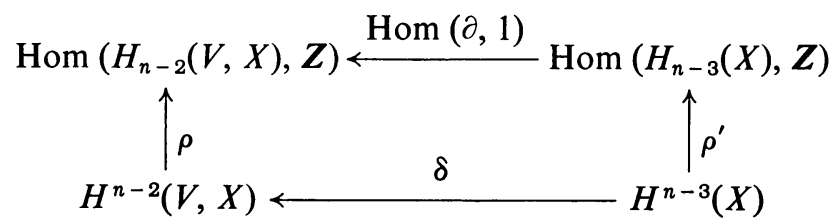

where $\rho$ and $\rho^{\prime}$ come from the Universal Coefficient theorem. It is not difficult to show that $0 \rightarrow H_{n-2}(V, X) \stackrel{\partial}{\rightarrow} H_{n-3}(X) \rightarrow H_{n-3}(V) \rightarrow 0$ is split exact and hence $\operatorname{Hom}(\partial, 1)$ is onto. But $\rho^{\prime}$ is onto and hence $\rho \delta$ is onto. But $H^{n-2}(V, X)$ is free, and hence $\rho$ is an isomorphism, and $\delta$ is onto. Using the facts that $H^{n-2}(V, X)$ is free and $\bar{h}^{*}=0$, it is not difficult to define $\alpha: H^{n-2}(V, X) \rightarrow H^{n-3}(X)$ such that $\delta \alpha=1$ and $\nu \alpha=0$. Now define $\alpha^{\prime}: H^{n-2}\left(V^{\prime}, \mathrm{Bd} N^{\prime}\right) \rightarrow H^{n-3}\left(\mathrm{Bd} N^{\prime}\right)$ by $\alpha^{\prime}=\nu^{\prime} \alpha\left(\varepsilon^{\prime}\right)^{-1}$. (Note that $\varepsilon^{\prime}$ is induced by an excision, so that $\alpha^{\prime}$ is well defined.) One easily checks that $\delta^{\prime} \alpha^{\prime}=1$ and im $\alpha^{\prime} \subset \nu^{\prime}(\operatorname{ker} \nu)$. It follows that im $\alpha^{\prime}$ is a free direct summand of $H^{n-3}\left(\operatorname{Bd} N^{\prime}\right)$. Now let $V_{1}, \ldots, V_{t}$ and $V_{1}^{\prime}, \ldots, V_{t}^{\prime}$ be the components of $V$ and $V^{\prime}$ respectively such that $V_{i}^{\prime} \subset V_{i}$. (Recall, $V=\mathrm{Cl}(U-N), V^{\prime}=\mathrm{Cl}\left(U-N^{\prime}\right)$, and $N^{\prime}$ satisfies $\mathscr{S}_{n-4}^{\prime}$ with respect to $N$.) Let $X_{i}=\mathrm{Cl}\left(V_{i}-V_{i}^{\prime}\right)$. Hence $V=\bigcup_{\text {dist }} V_{i}$, $V^{\prime}=\bigcup_{\text {disst }} V_{i}^{\prime}, \quad \operatorname{Bd} N=\operatorname{Bd} V=\bigcup_{\text {disst }} \operatorname{Bd} V_{i}, \quad \operatorname{Bd} N^{\prime}=\operatorname{Bd} V^{\prime}=\bigcup_{\text {disst }} \operatorname{Bd} V_{i}^{\prime}, \quad X=$ $\bigcup_{\text {disst }} X_{i}, \quad \operatorname{Bd} X_{i}=\mathrm{Bd} V_{i} \bigcup_{\text {disst }} \operatorname{Bd} V_{i}^{\prime}$. Let $\nu_{i}: \operatorname{Bd} V_{i} \subset X_{i}$ and $\nu_{i}^{\prime}: \operatorname{Bd} V_{i}^{\prime} \subset X_{i}$. It follows that, for $1 \leqq i \leqq t$, there is a commutative diagram

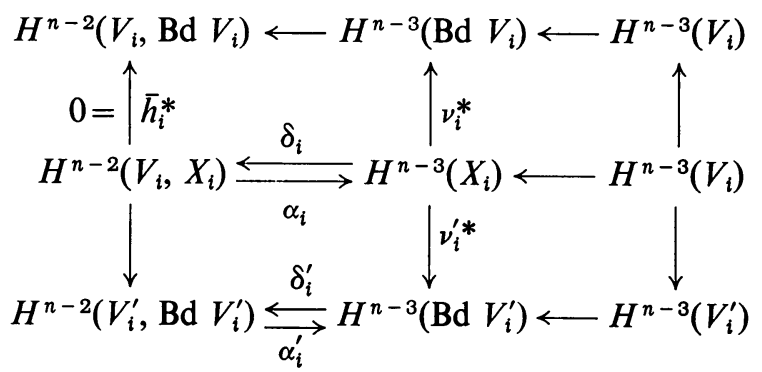


such that $\delta_{i} \alpha_{i}=1, \nu_{i}^{*} \alpha_{i}=0, \delta_{i}^{\prime} \alpha_{i}^{\prime}=1$, im $\alpha_{i}^{\prime} \subset \nu_{i}^{\prime *}\left(\operatorname{ker} \nu_{i}^{*}\right)$, and im $\alpha_{i}^{\prime}$ is a free direct summand of $H^{n-3}\left(\mathrm{Bd} V_{i}^{\prime}\right)$. By Lemma 11 of [2], the duality isomorphism

$$
\psi_{i}: H^{n-3}\left(\mathrm{Bd} V_{i}^{\prime}\right) \rightarrow H_{2}\left(\mathrm{Bd} V_{i}^{\prime}\right)
$$

sends $\nu_{i}^{\prime *}\left(\operatorname{ker} \nu_{i}^{*}\right)$ onto $\left(\operatorname{ker} \nu_{i *}^{\prime}\right)_{2}$. Hence $A_{i}=\psi_{i}\left(\mathrm{im} \alpha_{i}^{\prime}\right)$ is a free direct summand of $H_{2}\left(\operatorname{Bd} V_{i}^{\prime}\right)$. Let $\lambda_{1 i}, \lambda_{2 i}, \ldots, \lambda_{k_{i} i}$ be a basis for $A_{i} \subset H_{2}\left(\operatorname{Bd} V_{i}^{\prime}\right)$. Since each $\operatorname{Bd} V_{i}^{\prime}$ is 1-connected and $\operatorname{dim} \mathrm{Bd} V_{i}^{\prime}>4$, it follows that each $\lambda_{j i}$ can be represented by a PL embedded 2-sphere $S_{j i}$ such that $S_{j i} \cap S_{k l}=\varnothing$ unless $j=k$ and $i=l$. Since $A_{i} \subset\left(\operatorname{ker} \nu_{i *}^{\prime}\right)$ we can, using Irwin's embedding theorem, bound each $S_{j i}$ with a PL 3-ball $B_{j i}$ with Int $B_{j i} \subset$ Int $X_{i}$ such that $B_{j i} \cap B_{k l}=\varnothing$ unless $j=k$ and $i=l$. For each $B_{j i}$, take a regular neighborhood $M_{j i}$ such that $M_{j i} \cap M_{k l}=\varnothing$ unless $j=k$ and $i=l$.

Let $N_{n-3}=\mathrm{Cl}\left(N^{\prime}-\bigcup M_{j i}\right)$. We want to show that $N_{n-3}$ satisfies $\mathscr{S}_{n-3}$. Clearly (1) is satisfied since $N_{n-3} \supset N$ and Int $N \supset C$. One easily checks that the deletion of the handles and the surgery is such that, $\sigma \mathscr{C} \mathrm{Cl}\left(U-N_{n-3}\right)=\sigma \mathscr{C} \mathrm{Cl}\left(U-N^{\prime}\right)$ and $\sigma \mathscr{C} \mathrm{Bd} N_{n-3}=\sigma \mathscr{C} \mathrm{Bd} N^{\prime}$, and so (2) follows.

By finite induction, to show that each component of $\mathrm{Bd} N_{n-3}$ is 1-connected, it suffices to show that $\mathrm{Bd}\left(V_{i}^{\prime} \cup M_{j i}\right)$ is 1-connected. But $\mathrm{Bd} V_{i}^{\prime}$ is 1-connected and $\mathrm{Bd}\left(V_{i}^{\prime} \cup M_{j i}\right)$ is obtained from $\mathrm{Bd} V_{i}^{\prime}$ by surgery of index 2. So, using general position and the van Kampen theorem, it follows that $\mathrm{Bd}\left(V_{i}^{\prime} \cup M_{j i}\right)$ is 1-connected. Each component of $U-N_{n-3}$ is 1-connected, for by finite induction we may assume that such a component is $V_{i}^{\prime} \cup M_{j i}$ with $M_{j i} \approx B^{3} \times B^{n-3}$ and $V_{i}^{\prime} \cap M_{j i}$ $\approx S^{2} \times B^{n-3}$, and since $V_{i}^{\prime}$ is 1 -connected, so is $V_{i}^{\prime} \cup M_{j i}$ by the van Kampen theorem.

It remains to show (4); that $i: N_{n-3} \subset U$ induces isomorphisms $i_{*}: H_{r}\left(N_{n-3}\right)$ $\rightarrow H_{r}(U)$ for $r \leqq n-3$.

Let $\bar{V}_{i}=V_{i}^{\prime} \cup \bigcup_{j=1}^{k_{i}} M_{j i}, i=1, \ldots, t$ be the components of $\mathrm{Cl}\left(U-N_{n-3}\right)$. To show (4), it suffices to show that $H_{r}\left(\bar{V}_{i}, \mathrm{Bd} \bar{V}_{i}\right)=0$ for $r \leqq n-2$ and $i=1, \ldots, t$; for since $\bar{V}_{i} \cap \bar{V}_{j}=\varnothing$ if $i \neq j$, this will imply that $H_{r}\left(\cup \bar{V}_{i}, \cup \mathrm{Bd} \bar{V}_{i}\right)=0$. Hence by excision $H_{r}\left(U, N_{n-3}\right)=0$ for $r \leqq n-2$, which implies (4). By finite induction we may assume that $\bar{V}_{i}$ is obtained from $V_{i}^{\prime}$ by adjoining a single $M_{j i}$. Let $W_{j}=$ $\mathrm{Bd} V_{j}^{\prime}-\left(S^{2} \times\right.$ Int $\left.B^{n-3}\right)$ and consider the following commutative diagram where all maps are inclusions

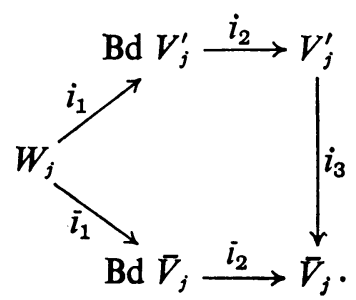

It now suffices to show the following:

(a) $\bar{i}_{2 *}: H_{r}\left(\mathrm{Bd} \bar{V}_{j}\right) \rightarrow H_{r}\left(\bar{V}_{j}\right)$ is an isomorphism for $r \neq 2$ but $r \leqq n-4$. 
(b) $\bar{i}_{2 *}: H_{2}\left(\mathrm{Bd} \bar{V}_{j}\right) \rightarrow H_{2}\left(\bar{V}_{j}\right)$ is an isomorphism.

(c) $H_{r}\left(\bar{V}_{j}, \mathrm{Bd} \bar{V}_{j}\right)=0$ for $r=n-3, n-2$.

To verify (a) it suffices to show that $i_{1}, i_{1}, i_{2}, i_{3}$ induce homology isomorphisms in dimension $k$ where $k \leqq n-4$ but $k \neq 2$. We will only check that $i_{1}$ induces isomorphisms; the arguments for the others being similar or easy. Let $T=S^{2} \times B^{n-3}$ and consider the reduced Mayer-Vietoris sequence for the triad $\left(\operatorname{Bd} V_{j}^{\prime} ; W_{j}, T\right)$.

$$
\begin{aligned}
\tilde{H}_{r}\left(S^{2} \times S^{n-4}\right) & \longrightarrow \tilde{H}_{r}\left(W_{j}\right) \oplus \tilde{H}_{r}\left(S^{2} \times B^{n-3}\right) \\
& \stackrel{\phi^{\prime}}{\longrightarrow} \tilde{H}_{r}\left(\mathrm{Bd} V_{j}^{\prime}\right) \stackrel{\phi}{\longrightarrow} \tilde{H}_{r-1}\left(S^{2} \times S^{n-4}\right) .
\end{aligned}
$$

If $r \neq 2$ but $r<n-4$, this sequence has the appearance $0 \rightarrow H_{2}\left(W_{j}\right) \rightarrow H_{r}\left(\mathrm{Bd} V_{j}^{\prime}\right) \stackrel{0}{\rightarrow}$ for we know that in this range $\phi$ is injective, since $S^{2} \times 0$ represents a free generator of $H_{2}\left(\mathrm{Bd} V_{j}^{\prime}\right)$, and hence $\phi^{\prime}=0$. Hence, in this range $i_{1 *}$ is an isomorphism. Now if $3 \leqq r=n-4$ the sequence appears as

$$
Z \stackrel{\alpha}{\longrightarrow} H_{n-4}\left(W_{j}\right) \stackrel{i_{1 *}}{\longrightarrow} H_{n-4}\left(\mathrm{Bd} V_{j}^{\prime}\right) \stackrel{\phi^{\prime}}{\longrightarrow} H_{n-5}\left(S^{2} \times S^{n-4}\right) \stackrel{\phi}{\longrightarrow} .
$$

As above $\phi^{\prime}=0$. Hence $i_{1 *}$ will be an isomorphism if $\alpha=0$. Notice that $\mathrm{Bd} W_{j}$ $=S^{2} \times S^{n-4}$ and consider the following commutative (up to sign) diagram where the isomorphisms are given by duality.

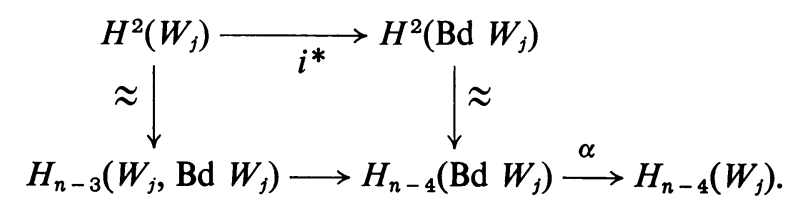

By exactness of the lower row, it now suffices to show that $i^{*}$ is surjective. But $i_{*}: H_{2}\left(\mathrm{Bd} W_{j}\right) \rightarrow H_{2}\left(W_{j}\right)$ carries the generator of $H_{2}\left(\mathrm{Bd} W_{j}\right)$ onto a free element of $H_{2}\left(W_{j}\right)$, since $S^{2} \times 0$ represents a primitive free element of a basis for $H_{2}\left(\mathrm{Bd} V_{j}^{\prime}\right)$. Hence there is a split short exact sequence of the form

$$
0 \longrightarrow H_{2}\left(\mathrm{Bd} W_{j}\right) \stackrel{i_{*}}{\longrightarrow} H_{2}\left(W_{j}\right) \longrightarrow G \longrightarrow 0 .
$$

Now consider the commutative diagram

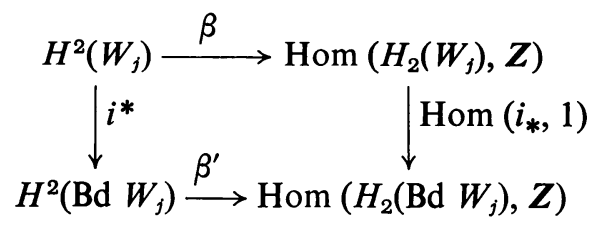

where $\beta$ and $\beta^{\prime}$ come from the Universal Coefficient theorem. It follows that $\beta$ and $\operatorname{Hom}\left(i_{*}, 1\right)$ are surjective. But $\beta^{\prime}$ is an isomorphism since $H^{2}\left(\operatorname{Bd} W_{j}\right)$ is free, and hence $i *$ is surjective. 
(b) follows, for as given in [2],

$$
\begin{aligned}
H_{2}\left(\operatorname{Bd} \bar{V}_{j}\right) & \approx H_{2}\left(\operatorname{Bd} V_{j}^{\prime}\right) / A_{j} \\
& \approx H_{2}\left(V_{j}^{\prime}\right) / \operatorname{inc}_{*}\left(A_{j}\right) \\
& \approx H_{2}\left(\bar{V}_{j}\right) .
\end{aligned}
$$

To verify (c), one first easily shows that $i_{2 *}$ is onto for dimensions less than $n-1$. Hence, using (a) and (b) and arguing as we did early in the proof of this step for $(V, \operatorname{Bd} N)=(V, \operatorname{Bd} V)$, it follows that $H_{r}\left(\bar{V}_{j}, \operatorname{Bd} \bar{V}_{j}\right)=0$ for $r \leqq n-3$ and

$$
H_{n-2}\left(\bar{V}_{j}, \operatorname{Bd} \bar{V}_{j}\right)
$$

is free. From this information, it follows, as it did for $\left(V_{j}^{\prime}, \mathrm{Bd} V_{j}^{\prime}\right)$, that the following is an exact sequence.

$$
0 \leftarrow H^{n-2}\left(\bar{V}_{j}, \mathrm{Bd} \bar{V}_{j}\right) \leftarrow H^{n-3}\left(\mathrm{Bd} \bar{V}_{j}\right) \leftarrow H^{n-3}\left(\bar{V}_{j}\right) \leftarrow 0 .
$$

By Poincaré duality, $H^{n-3}\left(\operatorname{Bd} \bar{V}_{j}\right) \approx H^{n-3}\left(\operatorname{Bd} V_{j}^{\prime}\right) / A_{j}^{\prime}$ where

$$
A_{j}^{\prime}=\alpha_{j}^{\prime}\left(H^{n-2}\left(V_{j}^{\prime}, \mathrm{Bd} V_{j}^{\prime}\right)\right) \text {. }
$$

But since $\alpha_{j}^{\prime}\left(\alpha_{j}^{\prime}\right.$ of the diagram on page 381) is a splitting homomorphism,

$$
H^{n-3}\left(\mathrm{Bd} \bar{V}_{j}\right) \approx H^{n-3}\left(V_{j}^{\prime}\right) .
$$

Now

$$
H^{n-3}\left(V_{j}^{\prime}\right) \approx \operatorname{Hom}\left(H_{n-3}\left(V_{j}^{\prime}\right), Z\right) \oplus \operatorname{Ext}\left(H_{n-4}\left(V_{j}^{\prime}\right), Z\right)
$$

and

$$
H^{n-3}\left(\bar{V}_{j}\right) \approx \operatorname{Hom}\left(H_{n-3}\left(\bar{V}_{j}\right), Z\right) \oplus \operatorname{Ext}\left(H_{n-4}\left(\bar{V}_{j}\right), Z\right) .
$$

But $H_{r}\left(V_{j}^{\prime}\right) \approx H_{r}\left(\bar{V}_{j}\right)$ for $r \neq 2$, and since $\operatorname{inc}_{*}\left(A_{j}\right)$ is a free direct summand of $H_{2}\left(V_{j}^{\prime}\right)$, from the argument given in (b) above, Ext $H_{2}\left(V_{j}^{\prime}\right) \approx$ Ext $H_{2}\left(\bar{V}_{j}\right)$. It follows that $H^{n-3}\left(\operatorname{Bd} \bar{V}_{j}\right) \approx H^{n-3}\left(\bar{V}_{j}\right)$. Since these groups are finitely generated and $H^{n-2}\left(\bar{V}_{j}\right.$, Bd $\left.\bar{V}_{j}\right)$ is free, the above exact sequence tells us that $H^{n-2}\left(\bar{V}_{j}\right.$, Bd $\left.\bar{V}_{j}\right)=0$. Now by the Universal Coefficient theorem, $H_{n-2}\left(\bar{V}_{j}, \mathrm{Bd} \bar{V}_{j}\right)=0$. This completes the proof of Proposition 3.1.

Proposition 3.2. If $C$ is a compact subset of the open connected PL n-manifold $U$ $(n>5)$ such that each component of $U-C$ is q-connected, then there is a compact connected PL n-submanifold $N$ of $U$ such that

(1) $C \subset$ Int $N$,

(2) $\sigma \mathscr{C} \mathrm{Bd} N=\sigma \mathscr{C}(U-N)=\sigma \mathscr{C}_{u}(U-C)$,

(3) each component of $\mathrm{Bd} N$ is $\min \{q,[(n-1) / 2]-1\}$-connected, and

(4) each component of $U-N$ is $\min \{q,[n / 2]-1\}$-connected.

Proof. For $0 \leqq i \leqq \min \{q,[n / 2]-1\}$ let $\mathscr{H}_{i}$ be the statement that there is a compact PL $n$-submanifold $N_{i}$ of $U$ such that (1), (2), (3), and (4) hold when $i$ and $N_{i}$ replace $q$ and $N$ respectively. If $q=0$, the techniques used in the proof of $\mathscr{S}_{1}$ of Proposition 
3.1 will prove Proposition 3.2; and if $q \geqq 1, \mathscr{H}_{1}$ is valid by the proof of $\mathscr{S}_{1}$. Now suppose that $\mathscr{H}_{i}$ is valid where $1 \leqq i<\min \{q,[n / 2]-1\}$. Let $M_{1}, M_{2}, \ldots, M_{t}$ be the components of $\mathrm{Bd} N_{i}$, and let $Q_{1}, Q_{2}, \ldots, Q_{t}$ be the components of $U-C$ such that $Q_{j} \supset M_{j}$. For each $j$, consider the triad $\left(Q_{j} ; M_{j}^{+}, \mathrm{Cl}\left(Q_{j}-M_{j}^{+}\right)\right)$where $M_{j}^{+}$ is the component of $\mathrm{Cl}\left(U-N_{i}\right)$ which contains $M_{j}$. Now consider the following portion of the resulting Mayer-Vietoris sequence

$$
H_{i+1}\left(M_{j}\right) \stackrel{\alpha}{\longrightarrow} H_{i+1}\left(M_{j}^{+}\right) \oplus H_{i+1}\left(\mathrm{Cl}\left(Q_{j}-M_{j}^{+}\right)\right) \longrightarrow H_{i+1}\left(Q_{j}\right) .
$$

Since $Q_{j}$ is $(i+1)$-connected, $\alpha$ is onto; and hence it follows that $H_{i+1}\left(M_{j}^{+}\right)$is finitely generated. Let $\lambda$ be a generator of $H_{i+1}\left(M_{j}^{+}\right)$. Choose $\mu \in H_{i+1}\left(M_{j}\right)$ such that $\alpha(\mu)=(\lambda, 0)$. Since $M_{j}$ is $i$-connected, let $f: S^{i+1} \rightarrow M_{j}$ be such that, via the Hurewicz isomorphism, $[f]=\mu$. By a general position argument, we may assume that $f$ is a PL embedding. Now $(\lambda, 0)=\alpha(\mu)=\alpha([f])=([f],-[f])$ and hence $[f]$ is trivial in $H_{i+1}\left(\mathrm{Cl}\left(Q-M_{j}^{+}\right)\right)$. But, since $M_{j}$ and $Q_{j}$ are both $i$-connected, so is $\mathrm{Cl}\left(Q_{j}-M_{j}^{+}\right)$, and hence $f$ is homotopically trivial in $\mathrm{Cl}\left(Q_{j}-M_{j}^{+}\right)$. Hence, we extend $f$ to $\bar{f}: B^{i+2} \rightarrow \mathrm{Cl}\left(Q_{j}-M_{j}^{+}\right)$. One easily verifies the hypothesis of Irwin's embedding theorem [23], and hence we may assume that $\bar{f}$ is a proper PL embedding. Let $P$ be a regular neighborhood of $\bar{f}\left(B^{i+2}\right)$ in $\mathrm{Cl}\left(Q_{j}-M_{j}^{+}\right)$, and let $N_{i+1}^{\prime}=$ $\mathrm{Cl}\left(N_{i}-P\right) . N_{i+1}^{\prime}$ will be our first approximation to $N_{i+1}$. Since $P \subset Q_{j} \subset U-C$ it is clear that $N_{i+1}^{\prime}$ satisfies (1). It is easy to see that neither the deletion of handles, nor surgery in this dimension range disconnects $N_{i}$ or any $M_{j}$, and hence $N_{i+1}^{\prime}$ satisfies (2).

Now let us see what happened to the connectivity conditions. Consider the triad $\left(M_{j}^{+} \cup P ; M_{j}^{+}, P\right)$. By Proposition 2.6 (3), $P \cap M_{j}^{+} \approx S^{i+1} \times B^{n-i-2}$. Now consider the reduced Mayer-Vietoris sequence

$$
\begin{aligned}
\tilde{H}_{k}\left(S^{i+1} \times B^{n-i-2}\right) \stackrel{\alpha_{k}}{\longrightarrow} \tilde{H}_{k}\left(M_{j}^{+}\right) \oplus \tilde{H}_{k}(P) \stackrel{\beta_{k}}{\longrightarrow} \tilde{H}_{k}\left(M_{j}^{+} \cup P\right) \\
\longrightarrow \tilde{H}_{k-1}\left(S^{i+1} \times B^{n-i-2}\right) .
\end{aligned}
$$

Note that $M_{j}^{+} \cup P \in \mathscr{C} \mathrm{Cl}\left(U-N_{i+1}^{\prime}\right)$ replaces $M_{j}^{+} \in \mathscr{C} \mathrm{Cl}\left(U-N_{i}\right)$, and what we wish to show is that $M_{j}^{+} \cup P$ is as nicely connected as $M_{j}^{+}$, and that in addition we have killed the generator $\lambda$ of $H_{i+1}\left(M_{j}^{+}\right)$. It is easy to show that $\tilde{H}_{k}\left(M_{j}^{+} \cup P\right)$ is flanked by zeros in the above sequence if $k \leqq i$, and using the van Kampen and Hurewicz theorems, it does follow that $M_{j}^{+} \cup P$ is $i$-connected. For $k=i+1$, $H_{k-1}\left(S^{i+1} \times B^{n-i-2}\right)=0$, and hence $\beta_{i+1}$ is surjective. It follows that

$$
H_{i+1}\left(M_{j}^{+}\right) / \operatorname{im} \alpha_{i+1} \approx H_{i+1}\left(M_{j}^{+} \cup P\right) .
$$

But $\left[\bar{f} \mid S^{i+1}\right]$ generates $H_{i+1}\left(S^{i+1} \times B^{n-i-2}\right)$ and $\alpha_{i+1}\left[\bar{f} \mid S^{i+1}\right]=[f]_{H_{i}+{ }_{1}\left(M_{j}{ }^{+}\right)}=\lambda$. Hence $H_{i+1}\left(M_{j}^{+} \cup P\right) \approx H_{i+1}\left(M_{j}^{+}\right) /(\lambda)$, and we have reduced $H_{i+1}\left(M_{j}^{+}\right)$by one generator. Hence we will be able to alter $N_{i}$ inductively to $N_{i+1}$ which satisfies (4) of $\mathscr{H}_{i+1}$, if we can show that $\mathrm{Bd}\left(M_{j}^{+} \cup P\right)$ is $\min \{i,[(n-1) / 2]-1\}$-connected. By Proposition 2.6, we obtained $\mathrm{Bd}\left(M_{j}^{+} \cup P\right)$ from $\mathrm{Bd} M_{j}^{+}=M_{j}$ by surgery of 
index $i+1$. Let $M_{j}^{\prime}=\mathrm{Bd}\left(M_{j}^{+} \cup P\right), T_{1} \approx S^{i+1} \times \operatorname{Int} B^{n-i-2}$ and $T_{2} \approx B^{i+2} \times S^{n-i-3}$, so that $M_{j}^{\prime}=\left(M_{j}-T_{1}\right) \cup T_{2}$ with $\left(M_{j}-T_{1}\right) \cap T_{2} \approx S^{i+1} \times S^{n-i-3}$. Consider the $\operatorname{triad}\left(M_{j}^{\prime} ; M_{j}-T_{1}, T_{2}\right)$ and the resulting reduced Mayer-Vietoris sequence,

$$
\tilde{H}_{k}\left(M_{j}-T_{1}\right) \oplus \tilde{H}_{k}\left(T_{2}\right) \rightarrow \tilde{H}_{k}\left(M_{j}^{\prime}\right) \rightarrow \tilde{H}_{k-1}\left(\left(M_{j}-T_{1}\right) \cap T_{2}\right) .
$$

For $k \leqq \min \{i,[(n-1) / 2]-1\} ; n-i-3>k$ and hence

$$
\tilde{H}_{k}\left(T_{2}\right)=0=\tilde{H}_{k-1}\left(\left(M_{j}-T_{1}\right) \cap T_{2}\right) \text {. }
$$

$M_{j}-T_{1}$ has the homotopy type of $M_{j}-S^{i+1}$ and since $M_{j}$ is $\min \{i,[(n-1) / 2]-1\}-$ connected, general position arguments give that $M_{j}-T_{1}$ is also

$$
\min \{i,[(n-1) / 2]-1\} \text {-connected. }
$$

It follows that $M_{j}^{\prime}$ is $\min \{i,[(n-1) / 2]-1\}$-connected.

It remains to show that (3) can be satisfied on the $i+1$ level. If $i+1 \geqq[(n-1) / 2]$, we are done; for any $M_{j}^{\prime} \in \mathscr{C} \mathrm{Bd} N_{i+1}^{\prime}$ is $\min \{i,[(n-1) / 2]-1\}$-connected. Now suppose $i+1<[(n-1) / 2]$. Let $\lambda$ be a generator of $\pi_{i+1}\left(M_{j}^{\prime}\right)$. Since we are in the trivial range, there is a PL embedding $f: S^{i+1} \rightarrow M_{j}^{\prime}$ such that $[f]=\lambda$. Since $i+1 \leqq[n / 2]-1$ and $M_{j}^{+} \cup P$ is $\min \{i+1,[n / 2]-1\}$-connected, we can extend $f$ to a proper PL embedding $\bar{f}: B^{i+2} \rightarrow M_{j}^{+} \cup P$. Let $P^{\prime}$ be a regular neighborhood of $\bar{f}\left(B^{i+2}\right)$ in $M_{j}^{+} \cup P$ and let $N_{i+1}=N_{i+1}^{\prime} \cup P^{\prime}$. By Proposition 2.6, we have obtained $M_{j}^{\prime \prime}=\mathrm{Bd} \mathrm{Cl}\left(\left(M_{j}^{+} \cup P\right)-P^{\prime}\right) \in \mathscr{C} \mathrm{Bd} N_{i+1}$ from $M_{j}^{\prime} \in \mathscr{C} \mathrm{Bd} N_{i+1}^{\prime}$ by surgery of index $i+1$. But, we are in the trivial range for surgery, and by Proposition 2.6, $\pi_{i+1}\left(M_{j}^{\prime \prime}\right) \approx \pi_{i+1}\left(M_{j}^{\prime}\right) /(\lambda)$. Hence the proposition will follow by induction, if we can show that $\left(M^{+} \cup P\right)-P^{\prime} \in \mathscr{C}\left(U-N_{i+1}\right)$ which replaces $M^{+} \cup P \in \mathscr{C}\left(U-N_{i+1}^{\prime}\right)$ is $\min \{i+1,[n / 2]-1\}$-connected, so that we will not destroy (4) at the $i+1$ level. But by Proposition 2.6, $P^{\prime} \approx \bar{f}\left(B^{i+2}\right) \times B^{n-i-2}$ and hence $\left(M_{j}^{+} \cup P\right)-P^{\prime}$ is of the homotopy type of $\left(M_{j}^{+} \cup P\right)-\bar{f}\left(B^{i+2}\right)$. Now using the connectivity of $M_{j}^{+} \cup P$, general position, and the fact that $i+1<[(n-1) / 2]$; the desired result follows easily.

Remark. Proposition 3.2 is an important step in proving the PL Hauptvermutung for the open PL manifolds that we consider, for it enables us to take a connectivity (at infinity) condition, which is not a priori related to any PL structure, and relate it to a given PL structure.

4. A connectivity characterization of $S^{n}(n>5)$ minus a nonempty tame compact 0-dimensional subset.

LEMMA 4.1. Let $U$ be an open $(p, q)$-connected PL $n$-manifold $(n>5)$ with $p \geqq[n / 2]$, $q \geqq 1$ and $p+q \geqq n-2$. Then $U$ is $(n-2)$-connected.

Proof. Using Proposition 3.2, it follows that $U$ is the monotone union of compact PL $n$-submanifolds $N_{i}$ such that

(1) Each component of $\mathrm{Bd} N_{i}$ is 1-connected.

(2) Each component of $U-N_{i}$ is $\min \{q,[n / 2]-1\}$-connected for each $i$.

(3) $\sigma \mathscr{C} \mathrm{Bd} N_{i}=\sigma \mathscr{C}\left(U-N_{\imath}\right)$.

(4) $N_{i}$ is 1-connected for each $i$. 
(1), (2), and (3) are immediate. To see (4) consider the decomposition $U=N_{i} \cup Q_{1 i}$ $\cup \cdots \cup Q_{r_{i} i}$ where the $Q_{j i}$ 's are the closures of the components of $U-N_{i}$. Since $U$ and each $Q_{j i} \cap N_{i}$ (a component of Bd $N_{i}$ ) are 1-connected, an application of the van Kampen theorem, a finite number of times, tells us that $N_{i}$ is 1-connected. Let $Q_{i}=\mathrm{Cl}\left(U-N_{i}\right)$. Now consider the exact reduced homology sequence for the pair $\left(U, Q_{i}\right)$

$$
\tilde{H}_{k}(U) \rightarrow H_{k}\left(U, Q_{i}\right) \rightarrow \tilde{H}_{k-1}\left(Q_{i}\right) \rightarrow \tilde{H}_{k-1}(U) .
$$

Since $U$ is $p$-connected and each component of $Q_{i}$ is $\min \{q,[n / 2]-1\}$-connected, it follows, using excision, that $H_{k}\left(N_{i}, \operatorname{Bd} N_{i}\right)=0$ if $2 \leqq k \leqq \min \{q+1,[n / 2]\}$ and $H_{1}\left(N_{i}, \mathrm{Bd} N_{\mathrm{i}}\right)$ is free. By duality and the Universal Coefficient theorem,

$$
\begin{aligned}
H_{r}\left(N_{i}\right) & \approx H^{n-r}\left(N_{i}, \operatorname{Bd} N_{i}\right) \\
& \approx \operatorname{Hom}\left(H_{n-r}\left(N_{i}, \operatorname{Bd} N_{i}\right), Z\right) \oplus \operatorname{Ext}\left(H_{n-r-1}\left(N_{i}, \operatorname{Bd} N_{i}\right), Z\right) .
\end{aligned}
$$

But $p+1 \leqq r \leqq n-2$ implies that $2 \leqq n-r \leqq \min \{q+1,[n / 2]\}$, and hence $H_{r}\left(N_{i}\right)=0$ for $p+1 \leqq r \leqq n-2$. Since $U$ is the monotone union of the $N_{i}$ 's, $H_{r}(U)=0$ for $p+1 \leqq r \leqq n-2$; and since $U$ is $p$-connected, $U$ is $(n-2)$-connected.

PROPOSITION 4.2. Let $U$ be an open $(p, q)$-connected PL $n$-manifold $(n>5)$ with $p \geqq[n / 2], q \geqq 1$ and $p+q \geqq n-2$. Let $C$ be a compact subset of $U$ such that each component of $U-C$ is 1-connected. Then there is a cored n-ball $C B_{k}^{n}$ such that $C \subset$ Int $C B_{k}^{n}$ and $k=\sigma \mathscr{C}_{u}(U-C)=\sigma \mathscr{C}\left(U-C B_{k}^{n}\right)$.

Proof. By Lemma 4.1, $U$ is $(n-2)$-connected. Hence $i: C \subset U$ induces a surjective homomorphism $i_{*}: H_{r}(C) \rightarrow H_{r}(U)$ for $r \leqq n-2$. Now let $N$ be a compact connected PL $n$-submanifold of $U$ which satisfies Proposition 3.1 for $C$. We will show that $N$ is the cored $n$-ball we are seeking. Note that $C \subset \operatorname{Int} N$ and $\sigma \mathscr{C} \operatorname{Bd} N=$ $\sigma \mathscr{C}_{u}(U-C)=\sigma \mathscr{C}(U-N)$ by Proposition 3.1. Also, $\tilde{H}_{r}(N)=0$ for $r \leqq n-3$. Hence $N$ will be $(n-3)$-connected, if $N$ is 1-connected. Let $Q_{1}, Q_{2} \ldots, Q_{t}$ be the components of $\mathrm{Cl}(U-N)$. Hence $U=N \cup Q_{1} \cup Q_{2} \cup \cdots \cup Q_{t}$ with each $N \cap Q_{t}$ being a component of $\mathrm{Bd} N$ and hence, 1-connected. Since $U$ is 1-connected, as we remarked in the proof of Lemma 4.1, $N$ is 1-connected. The proposition will follow from the following.

Claim. A compact $(n-3)$-connected PL $n$-manifold $N(n>5)$ with nonempty boundary such that each component of $\mathrm{Bd} N$ is 1-connected, is a cored $n$-ball.

Proof. By Lefschetz duality, $H_{n-r}(N, \mathrm{Bd} N) \approx H^{r}(N)$ and since $N$ is $(n-3)$ connected, $H^{r}(N)=0$ for $1 \leqq r \leqq n-3$. Hence, $H_{k}(N, \operatorname{Bd} N)=0$ for $3 \leqq k \leqq n-1$. Now consider the exact homology sequence for the pair $(N, \operatorname{Bd} N)$,

$$
H_{k}(N) \rightarrow H_{k}(N, \operatorname{Bd} N) \rightarrow H_{k-1}(\operatorname{Bd} N) \rightarrow H_{k-1}(N) .
$$

Since $N$ is $(n-3)$-connected, $H_{k}(N, \operatorname{Bd} N) \approx H_{k-1}(\operatorname{Bd} N)$ for $2 \leqq k \leqq n-3$; and hence $H_{r}(\mathrm{Bd} N)=0$ for $2 \leqq r \leqq n-4$. Since each component of $\mathrm{Bd} N$ is 1 -connected, it now follows that each component of $\mathrm{Bd} N$ is $(n-4)$-connected. But since $n \geqq 6$, 
$n-4 \geqq[(n-1) / 2]$ and $n-1 \geqq 5$. Hence, by a strong form of the Poincaré conjecture (essentially [17]), each component of Bd $N$ is a PL $(n-1)$-sphere. Let $S_{1}, S_{2}, \ldots, S_{m}$ be the components of $\mathrm{Bd} N$. Let $N^{\prime}$ be the closed PL $n$-manifold obtained from $N$ by inductively coning over the components of $\mathrm{Bd} N$, i.e.,

$$
N^{\prime}=N \cup C\left(S_{1}\right) \cup \cdots \cup C\left(S_{m}\right)
$$

where $C\left(S_{i}\right)$ is the cone over $S_{i}$ and $\mathrm{Cl}\left(N^{\prime}-C\left(S_{i}\right)\right) \cap C\left(S_{i}\right)=S_{i}$. By inductively applying the van Kampen theorem it is easy to see that $N^{\prime}$ is 1-connected. Suppose we have shown that $H_{r}\left(N^{\prime}\right)=0$ where $1 \leqq r<[n / 2]$. Consider the triad

$$
\left(N^{\prime} ; N, \cup C\left(S_{i}\right)\right)
$$

and the following portion of the resulting exact Mayer-Vietoris sequence,

$$
H_{r+1}(N) \oplus H_{r+1}\left(\bigcup C\left(S_{i}\right)\right) \rightarrow H_{r+1}\left(N^{\prime}\right) \rightarrow H_{r}\left(\bigcup S_{i}\right) .
$$

Since $n \geqq 6, n-3 \geqq[n / 2]$, and since $N$ is $(n-3)$-connected, $H_{r+1}(N)=0$. Since each $C\left(S_{i}\right)$ is a ball and $C\left(S_{i}\right) \cap C\left(S_{j}\right)=\varnothing$ if $i \neq j, H_{r+1}\left(\cup C\left(S_{i}\right)\right)=0$. Also $1 \leqq r<[n / 2]$ $<n-1$, and hence $H_{r}\left(\cup S_{i}\right)=0$. It follows that $H_{r+1}\left(N^{\prime}\right)=0$, and $N^{\prime}$ is [n/2]connected. Again by the Poincaré conjecture for spheres, $N^{\prime}$ is a PL $n$-sphere, and hence $N$ is a cored $n$-ball.

THeOREM 4.3. An open PL $n$-manifold $U(n>5)$ is PL homeomorphic to $S^{n}$ minus a nonempty tame compact 0 -dimensional subset $K$ of cardinality $\alpha$ if and only if there are positive integers $p$ and $q$ such that $p \geqq[n / 2], p+q \geqq n-2, U$ is $(p, q)$ connected, and $\mathscr{E} U$ has cardinality $\alpha$.

SuFfICIENCY. Since $U$ is 1 -connected at infinity, let $\left\{C_{i}\right\}$ be a sequence of compact subsets of $U$ such that $U=\bigcup$ Int $C_{i}, C_{i} \subset C_{i+1}$, and each component of $U-C_{i}$ is 1-connected for each $i$. Now by Proposition 4.2, $U$ is the monotone union of cored $n$-balls. By Theorem 2.2, $U$ is PL homeomorphic to $S^{n}-K$ for some nonempty tame compact 0 -dimensional set $K$. By Lemma 2.1 and the fact that homeomorphic manifolds have the same number of ends, $\sigma \mathscr{E} U=\sigma K$.

NECESSITY. Let $U \approx S^{n}-K$ where $K$ is a nonempty tame compact subset of cardinality $\alpha$. By Lemma 2.1, $\sigma K=\sigma \mathscr{E} U$. A theorem of McMillan [8], implies that $U$ is the monotone union of cored $n$-balls, and hence it follows easily that $U$ is $(n-2, n-2)$-connected.

REMARK. The conditions on the integers $p$ and $q$ in Theorem 4.3 cannot be made any weaker. Consider the following examples, all of which have exactly one end, but none of which are homeomorphic to $E^{n}$.

EXAMPLE 1. In [5], for $n \geqq 5$, contractible open PL $n$-manifolds which are not 1-connected at infinity are shown to exist.

Here $q \geqq 1$ fails. (Note that $p=\infty$.)

EXAMPLE 2. $\left(S^{[n / 2]} \times S^{[(n+1) / 2]}\right)$ minus a point is an $([n / 2]-1, n-2)$-connected open PL $n$-manifold which is not homeomorphic to $E^{n}$. Here $p \geqq[n / 2]$ just fails.

(Note that for $n \geqq 6,([n / 2]-1)+(n-2) \geqq n$.) 
EXAMPLE 3. For $n \geqq 7$ and $[n / 2] \leqq p \leqq n-4, S^{p+1} \times E^{n-p-1}$ is a $(p, n-p-3)$ connected open PL $n$-manifold which is not homeomorphic to $E^{n}$. Here $p+q$ $\geqq n-2$ just fails.

(Note that we need $n \geqq 7$ here, because for $n=6,[n / 2]+1=n-2$; and hence the theorem applies.)

\section{On the Hauptvermutung.}

THEOREM 5.1. Let $U$ and $U^{\prime}$ be homeomorphic open $(p, q)$-connected PL n-manifolds $(n>5)$ where $p$ and $q$ are two positive integers such that $p \geqq[n / 2], q \geqq 1$ and $p+q \geqq n-2$. Then $U$ and $U^{\prime}$ are PL homeomorphic.

Proof. Let $h: U \rightarrow U^{\prime}$ be a homeomorphism. Since $U$ is 1-connected at infinity, let $\left\{C_{i}\right\}_{i=1}^{\infty}$ be a sequence of compact subsets of $U$ such that each component of $U-C_{i}$ is 1-connected for each $i, C_{i} \subset \operatorname{Int} C_{i+1}$, and $\bigcup$ Int $C_{i}=U$. For each $i$, let $C_{i}^{\prime}=h\left(C_{i}\right)$. Let $M_{1}$ and $M_{1}^{\prime}$ be cored $n$-balls in $U$ and $U^{\prime}$ respectively which satisfy the conclusion of Proposition 4.2 with respect to $C_{1}$ and $C_{1}^{\prime}$ respectively. Hence $C_{1} \subset$ Int $M_{1}, C_{1}^{\prime} \subset$ Int $M_{1}^{\prime}$, and (index of $M_{1}$ ) $=\left(\right.$ index of $M_{1}^{\prime}$ ). Let $S_{11}, \ldots, S_{1 k_{1}}$ be the boundary spheres of $M_{1}$. Now let $S_{11}^{\prime}, \ldots, S_{1 k_{1}}^{\prime}$ be the boundary spheres of $M_{1}^{\prime}$ such that if $Q_{1 j}$ is the component of $U-C_{1}$ which contains $S_{1 j}$, then $S_{1 j}^{\prime} \subset h\left(Q_{1 j}\right)$. Let $g_{1}: M_{1} \rightarrow S^{n}$ be a PL embedding. Using Lemma 2.5 , it follows easily that there is a PL homeomorphism $g_{1}^{\prime}: M_{1}^{\prime} \rightarrow g_{1}\left(M_{1}\right)$ such that $g_{1}^{\prime}\left(S_{1 j}^{\prime}\right)=g_{1}\left(S_{1 j}\right)$ for $j=1, \ldots, k_{1}$. Let $h_{1}=\left(g_{1}^{\prime}\right)^{-1} g_{1}$. Hence $h_{1}$ is a PL homeomorphism of $M_{1}$ onto $M_{1}^{\prime}$ such that $h_{1}\left(S_{1 j}\right)=S_{1 j}^{\prime}$ for $j=1, \ldots, k_{1}$. Suppose now for $1 \leqq i \leqq r-1$, we have found a positive integer $s(i)$, cored $n$-balls $M_{i}$ and $M_{i}^{\prime}$ in $U$ and $U^{\prime}$ respectively, and a PL homeomorphism $h_{i}: M_{i} \rightarrow M_{i}^{\prime}$ such that

(1) $M_{i}$ and $M_{i}^{\prime}$ satisfy the conclusion of Proposition 4.2 for $C_{s(i)}$ and $C_{s(i)}^{\prime}$ respectively,

(2) Int $M_{i} \supset M_{i-1} \cup C_{i}$,

(3) Int $M_{i}^{\prime} \supset M_{i-1}^{\prime} \cup C_{i}^{\prime}$,

(4) $h_{i} \mid M_{i-1}=h_{i-1}$, and

(5) $h_{i}\left(S_{i j}\right)=S_{i j}^{\prime}, 1 \leqq j \leqq k_{i}$ where the $S_{i j}$ 's are the boundary spheres of $M_{i}$, and $S_{i j}^{\prime}$ is the boundary sphere of $M_{i}^{\prime}$ such that if $Q_{i j}$ is the component of $U-C_{s(i)}$ which contains $S_{i j}$, then $S_{i j}^{\prime} \subset h\left(Q_{i j}\right)$.

We wish to define $s(r), M_{r}, M_{r}^{\prime}$, and $h_{r}$. Since $U=\bigcup$ Int $C_{\mathfrak{i}}, U^{\prime}=\bigcup$ Int $C_{i}^{\prime}$, and $M_{r-1} \cup C_{r}, M_{r-1}^{\prime} \cup C_{r}^{\prime}$ are compact; choose an integer $s(r)$ such that Int $C_{s(r)}$ $\supset M_{r-1} \cup C_{r}$ and Int $C_{s(r)}^{\prime} \supset M_{r-1}^{\prime} \cup C_{r}^{\prime}$. Now apply Proposition 4.2 to $C_{s(r)}$ and $C_{s(r)}^{\prime}$, obtaining cored $n$-balls $M_{r}$ and $M_{r}^{\prime}$ such that $C_{s(r)} \subset$ Int $M_{r}$ and $C_{s(r)}^{\prime} \subset \operatorname{Int} M_{r}^{\prime}$. It follows that $M_{r}$ and $M_{r}^{\prime}$ have the same index. Let $S_{r 1}, \ldots, S_{r k_{r}}$ be the boundary spheres of $M_{r}$, and let $S_{r 1}^{\prime}, \ldots, S_{r k_{r}}^{\prime}$ be the boundary spheres of $M_{r}^{\prime}$ such that, if $Q_{r j}$ is the component of $U-C_{s(r)}$ which contains $S_{r j}$, then $S_{r j}^{\prime} \subset h\left(Q_{r j}\right)$. Clearly $M_{r}$ and $M_{r}^{\prime}$ satisfy (1), (2), and (3) for $i=r$. We must construct a PL homeomorphism 
$h_{r}: M_{r} \rightarrow M_{r}^{\prime}$ which satisfies (4) and (5). By Lemma 2.4, let $A_{r 1}, \ldots, A_{r k_{r-1}}, A_{r 1}^{\prime}, \ldots$, $A_{r k_{r-1}}^{\prime}$ be the pairwise disjoint cored $n$-balls such that

$$
\bigcup_{j=1}^{k_{r}-1} A_{r j}=\mathrm{Cl}\left(M_{r}-M_{r-1}\right), \quad \bigcup_{j=1}^{k_{r-1}} A_{r j}^{\prime}=\mathrm{Cl}\left(M_{r}^{\prime}-M_{r-1}^{\prime}\right),
$$

$A_{r j} \cap M_{r-1}=S_{(r-1) j}$, and $A_{r j}^{\prime} \cap M_{r-1}^{\prime}=S_{(r-1) j}^{\prime}$. It now suffices, to complete the inductive step, to define for $1 \leqq j \leqq k_{r-1}$, a PL homeomorphism $h_{r j}: A_{r j} \rightarrow A_{r j}^{\prime}$ such that

(a) $h_{r j}\left|S_{(r-1) j}=h_{r-1}\right| S_{(r-1) j}$ and

(b) $h_{r j}\left(S_{r m}\right)=S_{r m}^{\prime}$ if $S_{r m} \subset A_{r j}$.

For if we can do this, then

$$
\begin{aligned}
h_{r} & =h_{r-1} & & \text { on } M_{r-1}, \\
& =h_{r j} & & \text { on } A_{r j}
\end{aligned}
$$

will clearly satisfy (4) and (5). We will need the following.

Claim. $S_{r m} \subset A_{r j}$ if and only if $S_{r m}^{\prime} \subset A_{r j}^{\prime}$.

Proof. $S_{r m} \subset A_{r j}$ implies that $S_{r m} \subset Q_{(r-1) j}$; since $A_{r j} \cap M_{r-1}=S_{(r-1)\}}, Q_{(r-1) j}$ is a component of $U-C_{s(r-1)}$, and $A_{r j}$ is connected and lies in $U-C_{s(r-1)}$. By our construction, $S_{r m} \subset Q_{m}$. Hence since $Q_{(r-1) j} \in \mathscr{C}\left(U-C_{s(r-1)}\right), Q_{r m} \in \mathscr{C}\left(U-C_{s(r)}\right)$, and $C_{s(r-1)} \subset C_{s(r)}$; we have that $Q_{(r-1) j} \supset Q_{r m}$. Hence $h\left(Q_{(r-1) j}\right) \supset h\left(Q_{r m}\right) \supset S_{r m}^{\prime}$. But $S_{(r-1) j}^{\prime} \subset A_{r j}^{\prime} \cap h\left(Q_{(r-1) j}\right)$, and using the fact that $h\left(Q_{(r-1) j}\right)$ is a component of $C_{s(r-1)}^{\prime}$, we conclude that $A_{r j}^{\prime} \subset h\left(Q_{(r-1) j}\right)$. It follows that $A_{r k}^{\prime} \cap h\left(Q_{(r-1) j}\right)=\varnothing$ if $k \neq j$ and hence it must be that $S_{r m}^{\prime} \subset A_{r j}^{\prime}$.

Conversely, if $S_{r m} \notin A_{r j}$, then $S_{r m} \subset A_{r t}$ for $t \neq j$ since $S_{r m} \in \mathscr{C} \mathrm{Bd} M_{r}$ and

$$
\text { Bd } M_{r} \subset \bigcup_{k=1}^{k_{r}-1} \operatorname{Bd} A_{r k} \text {. }
$$

Hence by the first part $S_{r m}^{\prime} \subset A_{r t}^{\prime}$ and $A_{r t}^{\prime} \cap A_{r j}^{\prime}=\varnothing$. Hence $S_{r m}^{\prime} \phi A_{r t}^{\prime}$.

Now for $1 \leqq j \leqq k_{r-1}$, let $g_{r j}: A_{r j} \rightarrow S^{n}$ be a PL embedding. Hence

$$
\bar{g}_{r j}=g_{r j}\left(h_{r-1}^{-1} \mid S_{(r-1) j}^{\prime}\right)
$$

is a PL homeomorphism of $S_{(r-1) j}^{\prime}$ onto $g_{r j}\left(S_{(r-1) j}\right)$. By Lemma 2.5 and the claim, let $g_{r j}^{\prime}$ be an extension of $\bar{g}_{r j}$ to $A_{r j}^{\prime}$ such that $g_{r j}^{\prime}\left(S_{r m}^{\prime}\right)=g_{r j}\left(S_{r m}\right)$ whenever $S_{r m}^{\prime} \subset A_{r j}^{\prime}$. Now let $h_{r j}=\left(g_{r j}^{\prime}\right)^{-1} g_{r j}$. One easily verifies (a) and (b).

Now define $H: U \rightarrow U^{\prime}$ by $H(x)=h_{i}(x)$ whenever $x \in M_{i}$. It follows that $H$ is a PL homeomorphism.

In view of Theorem 4.3, we obtain the following corollary.

COROLlary. The Hauptvermutung holds for PL manifolds whenever the manifolds are topologically $S^{n}(n>5)$ minus a nonempty tame compact 0 -dimensional subset.

For example the manifolds may be topologically $S^{n}$ minus a tame Cantor set, $S^{n}$ minus any countable set, an open $n$-annulus, or $E^{n}$. (Stallings [19] has proven the corollary for $E^{n}(n \geqq 5)$.) 
THEOREM 5.2. For $n=6,7$ homeomorphic differentiable manifolds which are topologically $S^{n}$ minus a nonempty tame compact 0-dimensional subset are diffeomorphic.

Proof. Let $K$ be a nonempty tame compact 0-dimensional subset of $S^{n}$, and let $U$ and $V$ be differentiable manifolds which are homeomorphic to $S^{n}-K$. By [22], give $U$ and $V$ PL triangulations $T_{1}$ and $T_{2}$ respectively, which are compatible with their differentiable structures. By the preceding theorem, $\left(U, T_{1}\right)$ and $\left(V, T_{2}\right)$ are PL homeomorphic. By [8], $U$ is the monotone union of cored $n$-balls; and hence it follows that $\tilde{H}_{k}(U)=0$ if $k \neq n-1$ and $H_{n-1}(U)$ is free. It follows from [12], [4], [17], [7], and [9] that the least positive integer $a$ such that $\Gamma_{a} \neq 0$ is 7. For $n=6,7$; $H_{6}(U)$ is free and $H_{k}(U)=0$ for $k \geqq 7$. Now applying Theorem 6.5 of [12], it follows that $U$ and $V$ are diffeomorphic.

REMARK. Theorem 5.2 does not hold for arbitrary $n>5$. For let $\Sigma^{n}$ be an exotic $n$-sphere. Hence $\Sigma^{n} \times E^{1}$ is topologically an open $(n+1)$-annulus, but is not diffeomorphic to $S^{n} \times E^{1}$. For, if so, then $\Sigma^{n}$ can be smoothly embedded in $S^{n+1}$. Now by 3.6 of [11], $\Sigma^{n}$ is bicollared in $S^{n+1}$ and by [3], the closure of a component of $S^{n+1}-\Sigma$ is a topological ball $B$. By the smooth Hauptvermutung for balls [18]; $B$, as a smooth manifold, is diffeomorphic to $B^{n+1}$. Hence $\mathrm{Bd} B^{n+1}=\Sigma^{n}$ is diffeomorphic to $S^{n}$, contradicting the fact that $\Sigma^{n}$ is exotic, i.e., the existence of an exotic $n$-sphere implies the existence of an exotic open $(n+1)$-annulus.

\section{REFERENCES}

1. J. W. Alexander, The combinatorial theory of complexes, Ann. of Math. 31 (1930), 292-320.

2. W. Browder, J. Levine and G. R. Livesay, Finding a boundary for an open manifold, Amer. J. Math. 87 (1965), 1017-1028.

3. M. Brown, A proof of the generalized Schoenflies theorem, Bull. Amer. Math. Soc. 66 (1960), 74-76.

4. J. Cerf, Sur les difféomorphismes de la sphère de dimension trois $\left(\Gamma_{4}=0\right)$, Lecture Notes in Math. No. 53, Springer-Verlag, Berlin, 1968.

5. M. L. Curtis and K. W. Kwun, Infinite sums of manifolds, Topology 3 (1965), 31-42.

6. C. H. Edwards, Jr., Open 3-manifolds which are simply connected at infinity, Proc. Amer. Math. Soc. 14 (1963), 391-395.

7. M. Kervaire and J. Milnor, Groups of homotopy spheres, Ann. of Math. (3) 77 (1963), 504-537.

8. D. R. McMillan, Jr., Taming Cantor sets in $E^{n}$, Bull. Amer. Math. Soc. 70 (1964), 706-708.

9. J. Milnor, On manifolds homeomorphic to the 7-sphere, Ann. of Math. 64 (1956), 399-405.

10. - "A procedure for killing homotopy groups of differentiable manifolds" in Differential geometry, Proc. Sympos. Pure Math., Vol. 3, Amer. Math. Soc., Providence, R. I., 1961, pp. 39-55.

11. - Lectures on the h-cobordism theorem, Notes by L. Siebenmann and J. Sondow, Princeton Math. Notes, Princeton Univ. Press, Princeton, N. J., 1965.

12. J. R. Munkres, Obstructions to the smoothing of piecewise-differentiable homeomorphisms, Ann. of Math. 72 (1960), 521-544.

13. C. D. Papakyriakopoulos, A new proof of the invariance of the homology groups of a complex, Bull. Soc. Math. Grece 22 (1943), 1-154. 
14. T. Rado, Über den Begriff der Riemannschen Flache, Acta Univ. Szeged 2 (1924-1926), 101-121.

15. C. P. Rourke and B. J. Sanderson, Block bundles. I, Ann. of Math. (2) 87 (1968), 1-28.

16. L. C. Siebenmann, The obstruction to finding a boundary for an open manifold of dimension greater than five, Doctoral thesis, Princeton Univ., Princeton, N. J., 1965.

17. S. Smale, Generalized Poincare's conjecture in dimensions greater than four, Ann. of Math. 74 (1961), 391-406.

18. - Differentiable and combinatorial structures on manifolds, Ann. of Math. 74 (1961), 498-502.

19. J. R. Stallings, The piecewise linear structure of euclidean space, Proc. Cambridge Philos. Soc. 58 (1962), 481-488.

20. D. Sullivan, On the Hauptvermutung for manifolds, Bull. Amer. Math. Soc. 73 (1967), 598-600.

21. J. B. Waggoner, Producing PL homeomorphisms by surgery, Bull. Amer. Math. Soc. 73 (1967), 78-83.

22. J. H. C. Whitehead, On $C^{1}$-complexes, Ann. of Math. (2) 41 (1940), 809-824.

23. E. C. Zeeman, Seminar on combinatorial topology, Mimeographed notes, Inst. Hautes Etudes Sci., Paris, 1963.

University of TenNessee,

KNOXVILLE, TENNESSEE 